\section{Fluids in Small Confined Spaces Stirred with Self-Assembled Chains of Iron Beads}

Mixing in microfluidic channels is difficult because confined spaces inhibit viscosity-induced turbulence. Diffusive mixing can occur in these situations, but some devices utilizing submillimeter channels require faster mixing than this achieves. Pursuing stirring methods at the micrometer-scale is therefore a priority for lab-on-a-chip applications, and the most useful methods utilize the strong, long-range interaction between a magnet and a magnetic field. As reported in the July issue of Physical Review E (DOI: 10.1103/PhysRevE.80.016312; \#016312), J.E. Martin, L. Shea-Rohwer, and K.J. Solis of Sandia National Laboratories have introduced a method of mixing fluids with self-assembled chains of 4-7 $\mu \mathrm{m}$ carbonyl iron beads driven by a magnetic vortex field.

A vortex field is a combination of three fields, two perpendicular sinusoidal fields oscillating $90^{\circ}$ out of phase (a rotating field) and a third normal to their plane. By measuring the angular deflection due to the vortex magnetic field of $150 \mathrm{mg}$ of Fe particles in $2 \mathrm{ml}$ solution, the researchers measured the torque

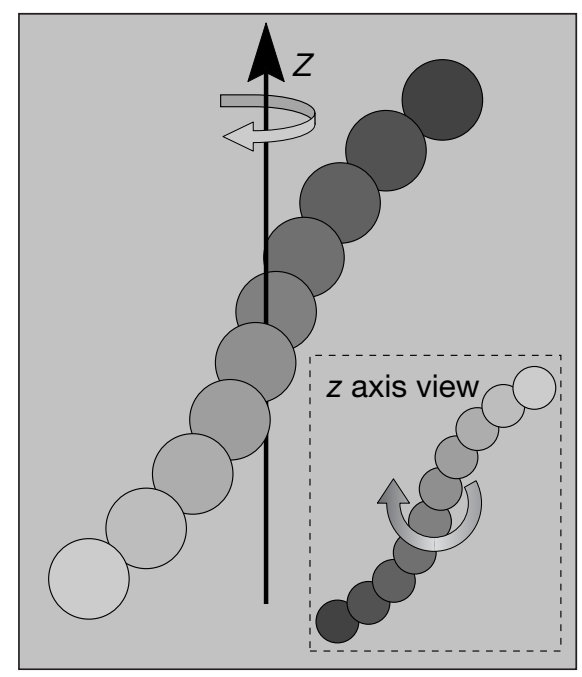

Figure 1. Magnetic beads rotate in response to a vortex field. Inset: The view along the mixing axis. Reprinted with permission from Physical Review $E$ 80 (2009), \#016312. (02009 by the American Physical Society.

imparted to several fluids with viscosities varying by an order of magnitude. They found that the mixing is optimized when the ac field and dc field magnitudes are equal. A sample of the magnetic powder was suspended in resin to test if the deflection measured was due to the magnetic torque of the beads or the torque imparted to the fluid. The resin did not move, from which the researchers inferred that the frequency-dependent susceptibility of the iron plays a negligible role.

The researchers found that the iron beads did not rotate independently; rather they stirred the liquids by forming magnetically linked chains (see Figure 1). Because chains fragment as they whirl around faster, the chain imparts a constant torque to the fluid as the frequency of the vortex field increases. This is not what happens with a magnetic stir-bar. The research team found that this action also keeps the mixing torque independent of the viscosity of the fluid and the diameter of the beads. It can be controlled only by changing the magnitude of the applied field. These effects ultimately cease at sufficiently high field frequencies or low field strengths that the chains fragment into solitary magnetic particles, but otherwise this method of imparting torque to a fluid allows for mixing fluids on any scale.

JIM RANTSCHLER

\section{Piezomagnetoelastic Device Harvests Vibrational Energy}

In the current era of energy awareness, the ability to recover energy wasted as heat or vibration is an important component of conservation. Commonly called "energy harvesting," it requires a way to gather and store minute amounts of energy, a complex problem that is leading researchers to novel materials solutions. As reported recently in Applied Physics Letters (DOI: 10.1063/1.3159815; \#254102), researchers A. Erturk, J. Hoffmann, and D.J. Inman, from Virginia Polytechnic Institute and State University, have devised a system for harvesting vibrational energy based on a magnetoelastic structure originally studied for the type of behavior known in chaos theory as strange attractor motion.

The device, a simple cantilevered construction with magnetic and piezoelectric components, achieves power generation over a broad spectrum of vibrational frequencies, which has been difficult to achieve but is important for effective energy harvesting. The design consists of a small cantilevered beam of a ferromagnetic material (tempered steel), the tip of which vibrates between two permanent rare earth magnets. Piezoceramic layers are used at the base of the beam to convert the vibration to electricity and the entire device is about $15 \mathrm{~cm}$ tall.

Erturk and colleagues present time domain simulations as well as experimental data, both demonstrating broad- band response achieved from a variety of starting conditions. They show that the presence and position of the permanent magnets near the beam tip is crucial. Without the magnets, the only significant output voltage occurs at the beam's resonant frequency whereas the full piezomagnetoelastic system delivers roughly $20 \mathrm{~V}$ across the investigated spectrum (4.5$8 \mathrm{~Hz}$ ). According to the researchers, their measured $200 \%$ increase in the open-circuit voltage translates into an $800 \%$ increase in the power amplitude, and they are optimistic that this design could be extended to other transduction mechanisms, such as electrostatic or magnetostrictive energy conversion.

ALISON HatT

\section{Soot Aggregates from Pre-Mixed Flame Observed to Have Low Fractal Dimension}

Fractal-like soot particles are among the combustion-generated carbonaceous aerosols that influence Earth's radiation balance, climate, atmospheric chemistry, and therefore the welfare of all its living inhabitants. In addition, fractal-like aggregates (FAs) are relevant to interstellar dust and to large-scale nanomaterial production with flame synthesis. Flameformation of aerosols and colloids in the cluster-dilute regime, where the average distance between clusters is much larger than the cluster size, occurs by a three- dimensional, diffusion-limited, clusteraggregation growth mechanism. For FAs formed by this mechanism, the mass is a function of linear size (i.e., the radius of gyration, $R_{\mathrm{g}}$ ) raised by an exponent, $D_{\mathrm{f}}$, which is the fractal dimension. Heretofore, FAs in the cluster-dilute regime have exhibited $D_{\mathrm{f}}$ values in the range of 1.7-1.8. 